\title{
MORE CHARACTERIZATIONS OF INNER PRODUCT SPACES
}

\section{J. T. JOICHI ${ }^{1}$}

Let $X$ be a real inner product space of dimension at least three and let $M$ be a 2-dimensional subspace of $X$. For a vector $u$ in $X$ but not in $M$, let $v$ be the vector in $M$ closest to $u$. It is easily seen that (i) if $v=0$, then all of the vectors of norm 1 in $M$ are equidistant from $u$ and (ii) if $v \neq 0$ and $w=|v|^{-1} v$, then of all the vectors of norm 1 in $M$ $w$ is the closest to $u$. The purpose of this paper is to show that each of these properties characterize those normed linear spaces which are inner product spaces. (For a survey of such results, see [3, pp. 115121].)

Throughout, we let $E$ denote real Euclidean 3-space. Our proofs are based on the following two characterizations of ellipsoids in $E$. Theorem A is due to G. Birkhoff [1]. Theorem B is due to Marchaud [4] and generalizes a result due to Blaschke [2, pp. 157-159].

(A) Let $K$ be a compact convex body in $E$ with bounding surface $S$. Suppose there exists a point 0 interior to $K$ satisfying: for any line $m$ through 0 and point $P$ in $m \cap S$, if $M$ is a plane through 0 so that its translate through $P$ supports $K$, then for skew cylindrical coordinates $(r, \theta, z)$ with $m$ the line $r=0$ and $M$ the plane $z=0$, the equation of $S$ is of the form $r=f(z) \cdot g(\theta)$. Then $K$ is an ellipsoid.

(B) Let $K$ be a compact convex body in $E$ with bounding surface $S$ satisfying: for every direction $d$ in $E$, there exists a corresponding plane $M_{d}$ such that the cylinder in the direction d generated by the plane curve $S \cap M_{d}$ circumscribes $K$. Then $K$ is an ellipsoid.

THEOREM. Let $X$ be a real normed linear space of dimension at least three. If $X$ satisfies either condition (1) or (2) below, then $X$ is an inner product space.

(1) For every 2-dimensional subspace $M$ of $X$ and vector $u$ not in $M$ for which $|u|=\min \{|u-w|: w$ in $M\}$, we have $|u-w|=\left|u-w^{\prime}\right|$ for all $w$ and $w^{\prime}$ in $M$ with $|w|=\left|w^{\prime}\right|=1$.

(2) For every 2-dimensional subspace $M$ of $X$ and vector $u$ not in $M$ for which there exists a vector $v$ in $M, v \neq 0$, satisfying $|u-v|$ $=\min \{|u-w|: w$ in $M\}$, we have

$$
\left.|u-| v\right|^{-1} v \mid=\min \{|u-w|: w \text { in } M,|w|=1\} .
$$

Received by the editors October 6,1966 and, in revised form, June 27,1967.

1 Research supported in part by the National Science Foundation under grant GP 5707. 
Proof. It suffices to show that for any 3-dimensional subspace $Y$ of $X$ and any one-to-one linear mapping of $Y$ onto $E$, the image $K$ of the unit ball in $Y$ is an ellipsoid (cf. [3]). For simplicity, we shall assume that $Y$ is $E$. The first of the above conclusions follows from Theorem $\mathrm{A}$ and the second from Theorem $\mathrm{B}$. The arguments are similar and we furnish only the latter.

Given a direction $d$, let $m$ be the line through 0 (the origin) in the direction $d$. Let $N$ be any plane containing $m$, let $n$ be a line in $N$ parallel to $m$ which supports $K \cap N$, and let $x$ be any point of $K \cap n$. Let $N^{\prime}$ be a plane parallel to $N$ which supports $K$ and let $y$ be any point of $K \cap N^{\prime}$. By the symmetry of $K$, the plane $N^{\prime \prime}$ parallel to $N$ and containing $-y$ will also support $K$. We wish to show that the 2-dimensional subspace $M$ of $E$ spanned by $x$ and $y$ has the desired property of $M_{d}$ in (B). Thus, for $S=$ boundary $K$, we need to show that for any point $z$ in $S \cap M$, the line $p$ through $z$ parallel to $m$ supports $K$. Suppose $z=a x+b y$ and $|z|=1$. If $a=0$ or $b=0$, then $z= \pm x$ or $\pm y$ and it is clear that $p$ has the desired property. Assume $a, b \neq 0$; by the symmetry of $K$, we need only consider $a>0$. Let $u=(1 / 2) x$ $-(b / 2 a) y$ and let

$$
K_{z}=\{w: w \text { in } E,|w-u| \geqq(1 / 2 a)\} .
$$

If $T$ is the mapping in $E$ defined by $T(w)=(1 / 2 a) w+u$, then $T(0)=u$, $T(K)=K_{z}$ and $T(z)=x$. Since $T$ is a magnification followed by a translation to show that $p$ supports $K$ at $z$, it suffices to show that $n$ supports $K_{z}$ at $x$.

We note that the ball centered at $u$ of radius $|b| / 2 a$ is supported by $N$ at $v=(1 / 2) x$. Thus, $v$ is in $K_{z}$ and by condition (2), $|u-x|$ $=\min \{|u-w|: w$ in $N,|w|=1\}$. Suppose $n$ does not support $K_{z}$. Then there must be a point $w_{0}$ common to $n$ and $i\left(K_{z}\right)$, the interior of $K_{z}$. It follows that all points of the segment [wo $v$ ], except possibly $v$, belong to $i\left(K_{z}\right)$. But the segment $\left[w_{0}, v\right]$ must contain a vector $w_{1}$ of norm 1 . We then have $\left|u-w_{1}\right|<|u-x|$, a contradiction. Thus, $n$ supports $K_{z}$ at $x$ and our conclusion follows.

\section{REFERENCES}

1. G. Birkhoff, Orthogonality in linear metric spaces, Duke Math. J. 1 (1935), 169-172. 1956.

2. W. Blaschke, Kreis und Kugel, Veit, Leipzig, 1916; 2nd ed., de Gruyter, Berlin,

3. M. M. Day, Normed linear spaces, Springer-Verlag, Berlin, 1958.

4. André Marchaud, Un thèoréme sur les corps convexes, Ann. Sci. École Norm. Sup. (3) 76 (1959), 283-304.

University of Minnesota 